\title{
Head-Group Acylation of Chloroplast Membrane Lipids
}

\author{
Yu Song ${ }^{1,2,+}$, Zolian S. Zoong Lwe ${ }^{1,2,+} \mathbb{D}$, Pallikonda Arachchige Dona Bashanee Vinusha Wickramasinghe ${ }^{2,3,+} \mathbb{C}$ \\ and Ruth Welti ${ }^{2,3, *(D)}$ \\ 1 Department of Biochemistry and Molecular Biophysics, Kansas State University, Manhattan, KS 66506, USA; \\ songyu@ksu.edu (Y.S.); zolian@ksu.edu (Z.S.Z.L.) \\ 2 Kansas Lipidomics Research Center, Kansas State University, Manhattan, KS 66506, USA; \\ vinuwickram@ksu.edu \\ 3 Division of Biology, Kansas State University, Manhattan, KS 66506, USA \\ * Correspondence: welti@ksu.edu \\ + These authors contributed equally to this work.
}

check for

updates

Citation: Song, Y.; Zoong Lwe, Z.S.; Wickramasinghe, P.A.D.B.V.; Welti, R. Head-Group Acylation of Chloroplast Membrane Lipids. Molecules 2021, 26, 1273. https://doi.org/10.3390/ molecules26051273

Academic Editor: Craig Frank

Received: 30 January 2021

Accepted: 24 February 2021

Published: 26 February 2021

Publisher's Note: MDPI stays neutral with regard to jurisdictional claims in published maps and institutional affiliations.

Copyright: (c) 2021 by the authors. Licensee MDPI, Basel, Switzerland. This article is an open access article distributed under the terms and conditions of the Creative Commons Attribution (CC BY) license (https:// creativecommons.org/licenses/by/ $4.0 /)$.
Abstract: Head group-acylated chloroplast lipids were discovered in the 1960s, but interest was renewed about 15 years ago with the discovery of Arabidopsides E and G, acylated monogalactosyldiacylglycerols with oxidized fatty acyl chains originally identified in Arabidopsis thaliana. Since then, plant biologists have applied the power of mass spectrometry to identify additional oxidized and non-oxidized chloroplast lipids and quantify their levels in response to biotic and abiotic stresses. The enzyme responsible for the head-group acylation of chloroplast lipids was identified as a cytosolic protein closely associated with the chloroplast outer membrane and christened acylated galactolipidassociated phospholipase 1 (AGAP1). Despite many advances, critical questions remain about the biological functions of AGAP1 and its head group-acylated products.

Keywords: Arabidopsis thaliana; plants; chloroplast lipids; plant stress; galactolipids; phosphatidylglycerol; head-group acylation; At2g42690; AGAP1

\section{Introduction}

Chloroplasts are organelles of plant cells, responsible for photosynthesis, storage of photosynthetic products including starch, and synthesis of primary and secondary metabolites, including fatty acids. Chloroplasts are surrounded by inner and outer membranes (collectively called the envelope) and, inside, contain stacks of thylakoid membranes, where the light reactions of photosynthesis occur. Chloroplast membrane lipids include the non-phosphorous-containing glycolipids monogalactosyldiacylglycerol (MGDG), digalactosyldiacylglycerol (DGDG), and sulfoquinovosyldiacylglycerol (SQDG), the phospholipid, phosphatidylglycerol (PG), and, in the envelope, phosphatidylcholine (PC) [1,2]. In addition, several classes of chloroplast lipids are formed primarily when plants are under stress, including polygalactosylated diacylglycerols [3-5], glucuronosyldiacylglycerols [6], and head-group acylated MGDGs, DGDGs, and PGs [7-10]. Here, we discuss our current knowledge of the head group-acylated chloroplast lipids, acylated MGDG (acMGDG), acylated DGDG (acDGDG), and acylated PG (acPG).

\section{Discovery of Head Group-Acylated Plastidic Lipids}

In 1967, Heinz reported the discovery of a glycolipid more hydrophobic than MGDG, with a 1:1:3 molar ratio of glycerol, galactose, and fatty acids in spinach leaf homogenates [11]. Soon a similar lipid was discovered in wheat flour [12]. The lipid from both sources was shown to be MGDG with a third acyl group esterified to the carbon at the 6-position of the galactose [12,13] (Figure 1a). 
<smiles>CC/C=C\C/C=C\C/C=C\CCCCCCCC(=O)OCC(C)OC1OC(COC(=O)CCCCCCCCCCCCCCC)C(O)C(O)C1O</smiles><smiles>CC/C=C\C/C=C\C/C=C\C/C=C/CCCCCCCC(=O)OCC1OC(OCC2OC(COCC(=O)OCCCCCCCCCCCCC)C(O)C(O)C2O)C(O)C(O)C1O</smiles>

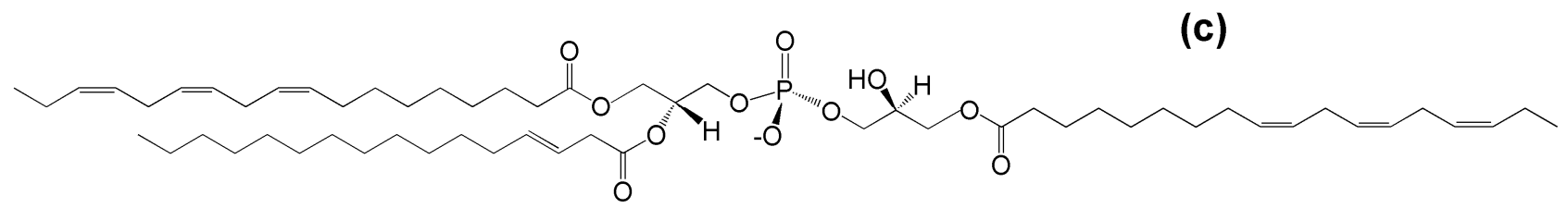

Figure 1. The molecular structures of (a) acylated monogalactosyldiacylglycerol (acMGDG), i.e., 1,2-di-O-acyl-3-O-(6'-Oacyl- $\beta$-D-galactopyranosyl)-sn-glycerol $\left(s n 1=18: 3^{\Delta 9,12,15}, s n 2=16: 3^{\Delta 7,10,13}, 6^{\prime}=16: 0\right),(\mathbf{b})$ acylated digalactosyldiacylglycerol (acDGDG), i.e., 1,2-di-O-acyl-3-O-[6'-O-(6"'-O-acyl- $\alpha$-D-galactopyranosyl)- $\beta$-D-galactopyranosyl) $]$-sn-glycerol ( $s n 1=s n 2=$ $6^{\prime \prime}=18: 3^{\Delta 9,12,15}$ ) and (c) acylated phosphatidylglycerol (acPG), i.e., 1-,2,di-O-acyl-sn-glycero-3-phospho-(3-acyl)-1-glycerol $\left(s n 1=18: 3^{\Delta 9,12,15}, s n 2=16: 1^{\Delta 3 \operatorname{trans}}, 3^{\prime}=18: 3^{\Delta 9,12,15}\right)$.

Later work by Heinz and colleagues identified acDGDG, which was found to have a fatty acid esterified to the 6-carbon of the second galactose [14] (Figure 1b). AcPG was identified in oats and later also found in Arabidopsis thaliana leaves [9,15] (Figure 1c). While extra-plastidic head-group acylated polar lipids, such as $\mathrm{N}$-acyl phosphatidylethanolamine, also have been identified in plants (as well as in animals), the focus of this review is the head-group acylated lipid classes of the plant chloroplast.

\section{Acylated Monogalactosyldiacylglycerol (AcMGDG) Is Ubiquitous in Photosynthetic Tissue}

AcMGDG species have been detected in green tissue of the major groups of land plants from non-vascular plants to angiosperms [16]. However, detected levels of acMGDG species after freeze-thawing vary considerably among plant species [16]. Outside the plant kingdom, only cyanobacteria and red algae have been reported to contain acMGDG species [17-19]. AcDGDG has been detected in spinach and Arabidopsis [14,20] and acPG has been found in oat seeds and Arabidopsis leaves $[9,15,20]$, but the presence of acPG and acDGDG elsewhere in the plant kingdom has not been systematically investigated. Similarly, while acylated lipids or the ability to produce them has been detected in oat seeds [15] and daffodil flowers [21], the presence of acylated chloroplast lipids in nonphotosynthetic tissues has not been systematically investigated.

\section{Lipidomics Is Increasing Our Knowledge of Head Group-Acylated Lipid Molecular Species}

The emergence of lipid analysis by electrospray ionization mass spectrometry, beginning around 2000, increased our ability to detect head group-acylated lipid molecular 
species present at low concentrations. It also provided the capability of analyzing larger numbers of biological samples. The structures of a limited number of head group-acylated lipid molecular species have been determined by nuclear magnetic resonance (NMR). As the number of detectable molecular species rapidly expanded with analysis by electrospray ionization mass spectrometry, accurate mass analysis of head group-acylated lipids and their fragments was used to identify the newly detected lipid molecular species at the level of chemical formula $[10,20,22]$. Ibrahim et al. used gas chromatography-mass spectrometry to carefully elucidate the structures of Arabidopsis fatty acids, then combined the fatty acid structural information with acylated lipid masses and fragmentation patterns detected by electrospray ionization mass spectrometry, to define acylated lipid structures [23]. In some cases, chromatography has been employed to provide separation before mass spectrometry $[9,23,24]$, while in other cases, plant extracts have been infused directly to the mass spectrometer $[10,20,22]$. Lower-resolution mass spectrometers, such as triple quadrupole instruments, employing precursor scanning or multiple reaction monitoring, often have been used for quantification. Caution is necessary in identification of acMGDG species in direct-infusion triple quadrupole mass spectrometry methods, because there are acMGDG molecular species that are isobaric or near isobaric (have the same or nearly the same mass).

In 2006, Andersson et al. [7] purified and used mass spectrometry and NMR to identify an acMGDG containing the jasmonate precursors, oxophytodienoic acid (OPDA) and dinor-OPDA (dnOPDA) (Figure 2). This lipid was named Arabidopside E, joining a series of "Arabidopsides", which are galactolipids that contain OPDA and dnOPDA [25-27]. Soon the same group discovered an all-OPDA version of acMGDG named Arabidopside G [8]. The observations of these Arabidopsis acMGDG molecular species containing multiple oxidized fatty acids fueled interest in a closer look at head group-acylated lipids in the chloroplast.

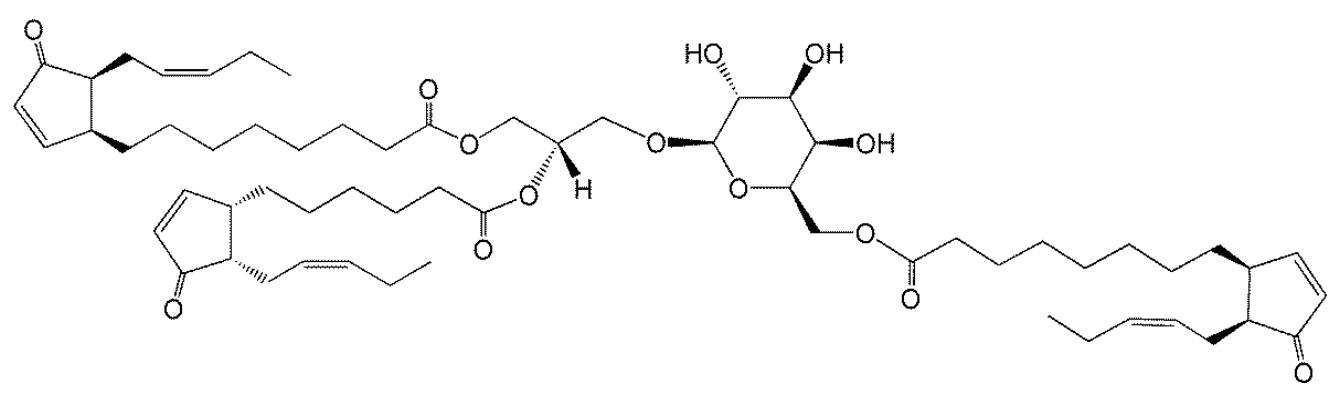

Figure 2. Arabidopside E, 1-O-OPDA,2-O-dnOPDA-3-O-(6'-O-OPDA- $\beta$-D-galactopyranosyl)-snglycerol, where OPDA and dnOPDA are abbreviations for oxophytodienoic acid and dinoroxophytodienoic acid, respectively.

To date, more than 100 acylated chloroplast lipid molecular species have been observed by mass spectrometry, and, in many cases, the molecular species have been quantified in relation to internal standards $[10,20,22,23,28]$. While the internal standards used typically are not well-matched to head group-acylated lipids in terms of structure, quantitation in relation to internal standards allows comparison of the levels of acylated lipids among samples.

In general, acMGDG is much more common than acDGDG or acPG. The most common fatty acids found in the head group of acMGDG are 18:3, 16:0, and 16:3 in "16:3 plants" and 18:3 and 16:0 in "18:3 plants" [10,16,29]. Other detectable non-oxidized fatty acids are 18:2, 18:1, and 18:0. Besides OPDA and dnOPDA, oxidized acyl chains with anion formulas $\mathrm{C}_{18} \mathrm{H}_{29} \mathrm{O}_{3}(18: 3 ; \mathrm{O}), \mathrm{C}_{18} \mathrm{H}_{29} \mathrm{O}_{4}(18: 3 ; \mathrm{O} 2)$, and several others have been detected in Arabidopsis acMGDG $[10,23]$. However, the prevalence of oxidized fatty acids in chloroplast lipids varies widely among plant species, with OPDA-containing lipids having a somewhat limited phylogenetic distribution. OPDA-containing lipids are found in Arabidopsis and some other Brassicaceae, and have been identified in a few species outside this family, 
including Melissa officinalis (lemon balm), Ipomoea tricolor (Mexican morning glory), and Cirsium arvense (creeping thistle) [16,30-32]. In sum, oxidized lipids, particularly OPDA and dnOPDA, are common in Arabidopsis, and these species are present in head group-acylated lipid species. However, the presence of OPDA and/or dnOPDA in head group-acylated chloroplast lipids has not been investigated in other species that contain these fatty acids.

\section{Varied Patterns of Acylated Chloroplast Lipids Are Induced by Environmental Stresses}

Biotic and abiotic stresses result in dramatic fluctuations in chloroplast lipid levels, with head-group acylation being strongly enhanced by stress (Figure 3). Whereas wounding and freeze-thaw treatments have been shown to increase the level of acylated lipids in a number of plant species, most studies of stress effects on chloroplast lipid acylation have been done in Arabidopsis thaliana $[7,8,10,16,20,22,23,28,33]$. The pattern of acylated chloroplast lipid accumulation varies as a function of the particular stress applied to Arabidopsis plants. Mechanical wounding, pathogen infection, freezing, and heat stress all induce head-group acylation of chloroplast lipids. In the photosynthetic tissue of Arabidopsis, which produces more oxidized fatty acid during stress than many other plants, patterns of lipids produced during various stresses differ in the amount of lipids acylated and in the relative amount of oxidized vs. non-oxidized acylated lipids produced (Figure 3). Strikingly, in Arabidopsis, oxidized lipid species, which make up only a few percent of chloroplast lipids when intact plants are subjected to wounding or pathogen infection [10], are concentrated in head group-acylated molecular species (Figure 3).

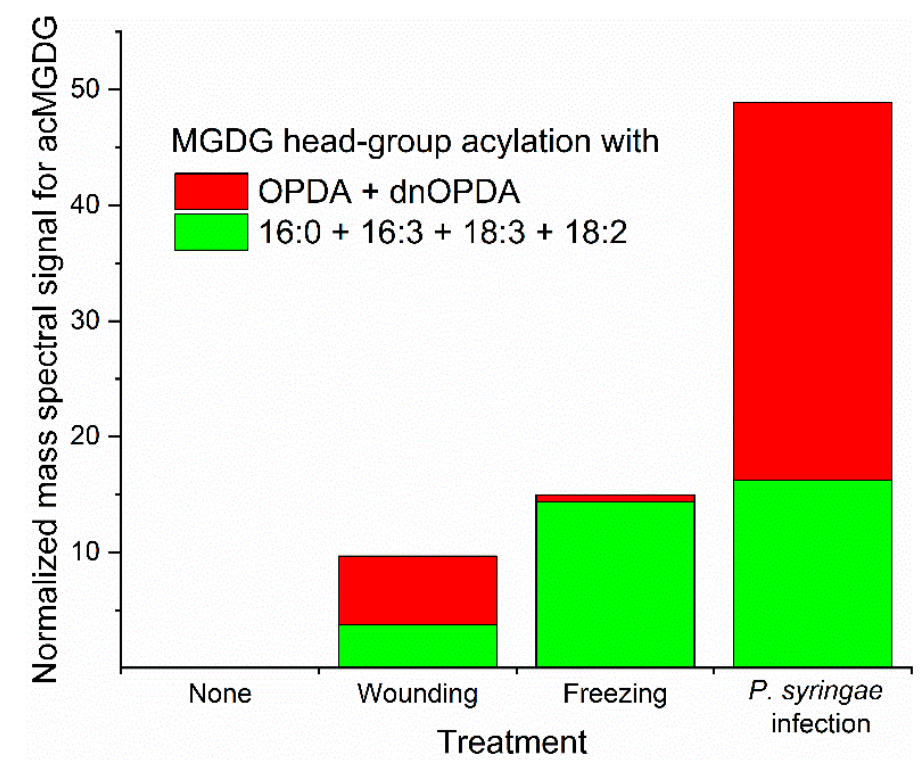

Figure 3. Arabidopsis thaliana accession Columbia-0 produces different acMGDG amounts and different molecular species when subjected to different stresses. Relative amounts of acMGDG headgroup acylation by non-oxidized $(16: 0+16: 3+18: 3+18: 2)$ (green) and oxidized (OPDA + dnOPDA) (red) on monogalactosyldiacylglycerol (MGDG) as a function of stress treatment. "Pseudomonas syringae infected" represents an avirulent infection at $24 \mathrm{~h}$. Freezing was $2 \mathrm{~h}$ at $-8{ }^{\circ} \mathrm{C}$ without acclimation and sampling after a $24 \mathrm{~h}$ recovery at $21^{\circ} \mathrm{C}$. Wounding was with a hemostat across the midvein and sampling $6 \mathrm{~h}$ later. Data are from reference 10.

When Arabidopsis (accession Columbia-0) is wounded, oxidation of membrane lipids is prominent, and levels of OPDA and dnOPDA increase in chloroplast lipids. Formation of acMGDGs, acDGDGs, and acPGs is induced by wounding [20]. Oxidized acMGDGs and non-oxidized acMGDGs are produced with different kinetics; oxidized acMGDGs are induced rapidly (levels are highest at $45 \mathrm{~min}$ and dropping $6 \mathrm{~h}$ after wounding), whereas non-oxidized acMGDGs are formed more slowly (levels are higher at $6 \mathrm{~h}$ than at 
45 min) $[10,20]$. AcDGDG analogs of Arabidopsides $E$ and $G$ and an OPDA-containing acPG are also induced during wounding, following a pattern similar to that of the oxidized acMGDGs. The metabolic basis of the different kinetics of oxidized and non-oxidized lipid species in wounding stress is unknown.

Bacterial infection of plants can be either virulent or avirulent. The virulent pathogen Pseudomonas syringae pv maculicola (Psm) causes great damage to plants, but relatively slight changes to the lipidome [22]. Recognition of bacterial effector protein AvrRpm1 secreted by an avirulent strain of $P$. syringae leads to a hypersensitive response and dramatic lipid changes [7,8]. Transgenic AvrRpm1 production results in massive accumulation of Arabidopside E, Arabidopside G, and accumulation of two OPDA-containing acPGs [7-9]. Both oxidized acMGDGs and non-oxidized acMGDGs are greatly induced after recognition of AvrRpm1 [10]. In a virulent infection, the levels of Arabidopside E were only $8 \%$ as high as during the avirulent interaction [22]. Interestingly, analysis of a mutant in allene oxide synthase, an enzyme involved in OPDA synthesis, showed that in the absence of fatty acyl oxidation, the hypersensitive response occurring in avirulent infection still results in formation of acMGDGs, but mainly non-oxidized species [9].

Hot and cold temperatures also stress plants, resulting in the production of head-group acylated chloroplast lipids in Arabidopsis leaves. Sub-lethal freezing of Arabidopsis induces acMGDGs in a different accumulation pattern compared to wounding and pathogen infection (Figure 3). In freezing, levels of non-oxidized acMGDGs were significantly increased during the post-freezing period, while levels of oxidized acMGDGs were very low [10]. Strikingly, at $24 \mathrm{~h}$ after freezing, Arabidopsis plants that had been acclimated at $4{ }^{\circ} \mathrm{C}$ before freezing had 5 -fold less acMGDGs than plants frozen without the $4{ }^{\circ} \mathrm{C}$ pre-treatment [10]. While, in general, increases of head group-acylated chloroplast lipids in heating stress are not as large as in avirulent bacterial interaction, wounding, or freezing stress, both non-oxidized and oxidized acMGDGs increase significantly when plants are subjected to heating stress $[28,33]$.

\section{AcMGDG Is Produced by Transacylation}

Heinz's early work demonstrated that the ability of spinach homogenates to produce acMGDG was destroyed by boiling, indicating that an enzyme was required for formation of the acylated lipid [11]. Incubating a protein extract of spinach leaves, at acidic $\mathrm{pH}$, with purified MGDG resulted in synthesis of acMGDG, while MGDG levels dropped [34]. Data indicate that acMGDG is formed mainly by acyl transfer of a glycerol-linked fatty acid in DGDG to the galactose of MGDG, producing digalactosylmonoacylglycerol (DGMG) in addition to the acMGDG (Equation (1)). To a lesser extent, a glycerol-linked fatty acid can be transferred from one MGDG to the galactose of another MGDG, producing monogalactosylmonoacylglycerol (MGMG) in addition to the acMGDG, in a reaction that Heinz called dismutation (Equation (2)) [34]. There is only a slight positional specificity with regard to the fatty acyl chain that is transferred, with the fatty acyl chain in the sn-1 position being slightly favored [35]. Additionally, transfer of an acyl chain from a phospholipid donor has been observed, but phospholipids are less favored as donors compared to galactolipids [34].

$$
\begin{gathered}
\text { DGDG + MGDG } \rightarrow \text { acMGDG + DGMG } \\
2 \mathrm{MGDG} \rightarrow \text { acMGDG + MGMG }
\end{gathered}
$$

\section{The Enzymatic Activity Catalyzing Head-Group Acylation of Chloroplast Lipids}

Heinz, who initially identified acMGDG in spinach leaves, purified the MGDG acylating activity from leaves of broad beans (Vicia faba), determining that the enzymatic activity is highest at $40^{\circ} \mathrm{C}$ and at pH 5.4 [36]. Nilsson and coworkers took up the quest to identify the enzyme responsible for formation of head group-acylated lipids in the chloroplast [16]. Based on their finding that acMGDG accumulated in large amounts in oat leaves after freeze-thaw treatment and considering the enzyme properties identified by Heinz, they 
purified the putative MGDG acyltransferase from oat leaves. A Basic Local Alignment Search Tool (BLAST) search of the amino acid sequence of the oat leaf protein against the Arabidopsis genome identified the gene At2g42690. Expression of At2g42690 in E. coli and in vitro analysis of the purified protein provided strong evidence for its function in catalyzing acMGDG formation. Given that At2g42690 belongs to a family of phospholipases and is tightly linked to the formation of head group-acylated galactolipids, the authors proposed the name acylated galactolipid-associated phospholipase 1 (AGAP1) for the enzyme responsible for acMGDG formation. Two Arabidopsis T-DNA insertion mutant lines, agap1-1 and agap1-2, both failed to accumulate wild-type levels of acMGDG, and also acPG and acDGDG, after freeze-thaw treatment, indicating that AGAP1 is responsible for the acylation of all three lipids [16]. However, small amounts of acMGDG, acPG, and acDGDG are present in the agap1 mutants and may be produced by related gene products.

\section{Sub-Cellular Localization of Acylated Galactolipid-Associated Phospholipase 1 (AGAP1) and Head Group-Acylated Chloroplast Lipids}

Heinz, Heemskerk, and colleagues found that the spinach MGDG-acylating activity was present in isolated outer chloroplast membranes from spinach leaves [37,38]. Based on the lack of a transit peptide and observed localization of a fluorescent tagged version of At2g42690 transiently expressed in tobacco, Nilsson et al. concluded that AGAP1 most likely resides in the plant cytosol and acts on galactolipids at the outer membrane of the chloroplast or on membrane fragments released by broken chloroplasts [16].

It is worth noting that in the Columbia- 0 accession of Arabidopsis the most common molecular species, Arabidopsides E and G, which contain only OPDA or dnOPDA chains, account for $40-50 \%$ of the total head group-acylated chloroplast lipids produced in vivo after mechanical wounding or bacterial infection, although the percentage of OPDA in MGDG and DGDG is only approximately $1-2 \%$ (Figure 3) [10]. This may imply that the OPDA-containing galactolipids that likely serve as precursors to Arabidopsides E and G are sequestered in the location where head-group acylation occurs, away from the bulk nonoxidized MGDG and DGDG. Formation of OPDA from linolenic acid (18:3) in galactolipids occurs on the esterified chains (the intact galactolipid) rather than on free fatty acids [39]. A key enzyme leading to OPDA, allene oxide synthase, has been localized to the chloroplast envelope (inner and / or outer membranes) by several groups [40-43], although it has also been associated with the thylakoid membranes [44] and plastoglobules [45]. Based on the majority of these data, it could be speculated that the highly oxidized head-group acylated molecular species formed under stress are located in the envelope, and that the oxidation and acylation pathways are co-localized. Furthermore, it could be implied that the lipids undergoing oxidation and acylation reactions are not readily mixing with the bulk chloroplast lipids, either due to physical separation or substrate channeling among stressresponsive, lipid-metabolizing enzymes. Distinct localization might also affect catabolic enzyme access to head group-acylated lipids, leading to the observed shorter lives of oxidized vs. non-oxidized acylated molecular species [20], but all hypotheses related to the localization of acylation and acylated lipids remain to be tested.

\section{Little Is Known about the Physical Properties of Head Group-Acylated Chloroplasts Lipids}

Virtually nothing has been reported on the physical properties of chloroplast head group-acylated lipids. It is well-established that DGDG is a bilayer-forming lipid, while MGDG's head group is small, and it tends to form inverted hexagonal phase, rather than a bilayer, unless its properties are modulated by the other bilayer components. These properties are similar to those of the phospholipids, PC, which is bilayer-forming, and phosphatidylethanolamine, which tends to form an inverted hexagonal phase. Although no studies have been undertaken on head group-acylated chloroplast lipids, there is analysis of the structure of $\mathrm{N}$-acylated phosphatidylethanolamine [46]. This lipid's third acyl chain is fully embedded in the membrane to a degree similar to the acyl chain in the sn2-position on the glycerol backbone. The $\mathrm{N}$-acylation of phosphatidylethanolamine with 
a saturated or mono-unsaturated fatty acid results in only modest changes in the phase transition temperature of the membrane and interaction with other membrane components, compared to non-acylated phosphatidylethanolamines with the same acyl chains [47]. However, it seems possible that the situation could be different for acMGDG, given that the head-group acylation is likely to be either with a third polyunsaturated fatty acid, which has a high cross-sectional area, or even a third oxidized fatty acid, such as OPDA, which has a very high cross-sectional area, as well as a shorter length due to the presence of the cyclopentenone ring. Such head group acylation, along with the production of monoacyl lipid molecular species by AGAP1, could potentially affect bilayer stability. Galactolipids with multiple oxidized fatty acids also might have limited miscibility with other lipids in the membrane. Another possibility is that acylated molecular species such as Arabidopsides E and G could have significant water solubility that might allow them to move into the aqueous phase more readily than molecular species with non-oxidized fatty acids. At this juncture, this is speculation.

\section{The Biological Functions of AGAP1 and Chloroplast Lipid Head-Group Acylation Remain Elusive}

Despite ample evidence that acylated galactolipids (oxidized and non-oxidized) accumulate in response to biotic and abiotic stresses, the biological functions of AGAP1 and head group-acylated chloroplast lipids remain elusive $[7,8,10,16]$. Both agap1 mutants appear to be unaffected by their defect in acylated lipid production under standard growth conditions. Here we consider three hypotheses about the function of AGAP1 and the lipids it acylates.

Hypothesis 1. High levels of head group-acylated lipids produced in pathogen response or wounding help plants survive the stresses. Arabidopside E has been demonstrated to have slight bactericidal activity against Pseudomonas syringae [7], and both Arabidopsides E and G somewhat reduce growth of the necrotrophic fungus Botrytis cinerea, while free OPDA has no effect [8]. Despite the anti-pathogen effects of the tested acMGDGs, Nilsson et al. [16] found that the agap1-1 mutant did not differ much from wild type in its ability to produce a hypersensitive response to $P$. syringae expressing an avirulent effector or in the ability of the bacteria to grow in their leaves. The authors concluded that AGAP1 and head group-acylated lipids formed during bacterial infection likely do not have a major role in the outcome of plants responding to pathogens via the hypersensitive response. The agap1-1 mutant and wild-type plants were also similar in their ability to support the feeding and growth of cotton leafworms (Spodoptera littoralis).

Hypothesis 2. The function of head group-acylated galactolipids containing OPDA and $d n O P D A$ is to serve as phytohormone reservoirs that could result in faster or larger responses in jasmonates if the plants encounter a second stimulus. Vu and coworkers tested this hypothesis by rewounding Arabidopsis leaves 24 or $48 \mathrm{~h}$ after a first wounding [10]. Although the levels of acMGDGs were higher after the second wounding event, the levels of free OPDA and jasmonic acid were not significantly higher nor did they appear more quickly after rewounding.

Hypothesis 3. AGAP1 and head group-acylated chloroplast lipids play a role in sequestration of acyl chains during an enhanced period of acyl-chain turnover caused by biotic or abiotic stress. This hypothesis suggests that AGAP1 might play a role analogous to that proposed for another transacylase, phospholipid:diacylglycerol acyltransferase 1 (PDAT1), which transfers an acyl chain from PC to diacylglycerol to produce triacylglycerol and lysoPC. Mueller et al. [33] demonstrated that Arabidopsis PDAT1 contributes to basal thermotolerance by sequestering displaced fatty acids in triacylglycerol during heat stress. Thus, one possibility is that the ability of AGAP1's transacylation activity to remove acyl chains from the membrane under stress conditions helps plants during or after stress. This remains to be tested.

Nilsson et al. [16] point out that the presence of head group-acylated chloroplast lipids across the plant kingdom and their maintenance through considerable evolutionary time 
suggests that they have a biological role. They also suggest that the role of AGAP1 and head group-acylated chloroplast lipids likely lies in realm of response to biotic and abiotic stresses that damage the chloroplast. We concur and suggest that characterization of the physical properties of the head group-acylated lipids, further work on their subcellular location, and continued analysis of AGAP1 mutants may shed light on the role of AGAP1 and the ubiquitous modification of chloroplast lipids by head-group acylation.

Author Contributions: Conceptualization, Y.S., Z.S.Z.L., P.A.D.B.V.W. and R.W.; writing-original draft preparation, Y.S., Z.S.Z.L. and P.A.D.B.V.W.; writing-review and editing, Y.S., Z.S.Z.L., P.A.D.B.V.W. and R.W. All authors have read and agreed to the published version of the manuscript.

Funding: The authors are grateful to the Johnson Cancer Research Center for a summer stipend award to Y.S. and for support from the USDA National Institute of Food and Agriculture (Hatch/Multi-State project 1013013). Contribution no. 21-204-J from the Kansas Agricultural Experiment Station.

Institutional Review Board Statement: Not applicable.

Informed Consent Statement: Not applicable.

Acknowledgments: The authors appreciate the helpful comments of Pamela Tamura and also are grateful for the reviewers' comments.

Conflicts of Interest: The authors declare no conflict of interest.

\section{References}

1. Uemura, M.; Steponkus, P.L. Effect of cold acclimation on the lipid composition of the inner and outer membrane of the chloroplast envelope isolated from rye leaves. Plant Physiol. 1997, 114, 1493-1500. [CrossRef] [PubMed]

2. Hölzl, G.; Dörmann, P. Chloroplast lipids and their biosynthesis. Annu. Rev. Plant Biol. 2019, 70, 51-81. [CrossRef]

3. Moellering, E.R.; Muthan, B.; Benning, C. Freezing tolerance in plants requires lipid remodeling at the outer chloroplast membrane. Science 2010, 330, 226-228. [CrossRef] [PubMed]

4. Moellering, E.R.; Benning, C. Galactoglycerolipid metabolism under stress: A time for remodeling. Trends Plant Sci. 2010, 16, 98-107. [CrossRef]

5. Vu, H.S.; Roston, R.; Shiva, S.; Hur, M.; Wurtele, E.S.; Wang, X.; Shah, J.; Welti, R. Modifications of membrane lipids in response to wounding of Arabidopsis thaliana leaves. Plant Signal. Behav. 2015, 10, e1056422. [CrossRef] [PubMed]

6. Okazaki, Y.; Otsuki, H.; Narisawa, T.; Kobayashi, M.; Sawai, S.; Kamide, Y.; Kusano, M.; Aoki, T.; Hirai, M.Y.; Saito, K. A new class of plant lipid is essential for protection against phosphorus depletion. Nat. Commun. 2013, 4, 1510. [CrossRef]

7. Andersson, M.X.; Hamberg, M.; Kourtchenko, O.; Brunnström, Å.; McPhail, K.L.; Gerwick, W.H.; Göbel, C.; Feussner, I.; Ellerström, M. Oxylipin profiling of the hypersensitive response in Arabidopsis thaliana: Formation of a novel oxo-phytodienoic acid-containing galactolipid, Arabidopside E. J. Biol. Chem. 2006, 281, 31528-31537.

8. Kourtchenko, O.; Andersson, M.X.; Hamberg, M.; Brunnström, Å.; Göbel, C.; McPhail, K.L.; Gerwick, W.H.; Feussner, I.; Ellerström, M. Oxo-phytodienoic acid-containing galactolipids in Arabidopsis: Jasmonate signaling dependence. Plant Physiol. 2007, 145, 1658-1669. [CrossRef] [PubMed]

9. Nilsson, A.K.; Johansson, O.N.; Fahlberg, P.; Steinhart, F.; Gustavsson, M.B.; Ellerström, M.; Andersson, M.X. Formation of Oxidized phosphatidylinositol and 12-oxo-phytodienoic acid containing acylated phosphatidylglycerol during the hypersensitive response in Arabidopsis. Phytochemistry 2014, 101, 65-75. [CrossRef]

10. Vu, H.S.; Roth, M.R.; Tamura, P.; Samarakoon, T.; Shiva, S.; Honey, S.; Lowe, K.; Schmelz, E.A.; Williams, T.D.; Welti, R. Headgroup acylation of monogalactosyldiacylglycerol is a common stress response, and the acyl-galactose acyl composition varies with the plant species and applied stress. Physiol. Plant. 2014, 150, 517-528. [CrossRef]

11. Heinz, E. Acylgalactosyldiglycerides from leaf homogenates. Biochim. Biophys. Acta 1967, 144, 321-332. [CrossRef]

12. Myhre, D.V. Glycolipids of Soft Wheat Flour. I. Isolation and characterization of 1-O-(6-O-acyl- $\beta$-D-galactopyranosyl)-2,3-di-Oacyl-D-glyceritols and phytosteryl 6-O-acyl-B-D-glucopyranosides. Can. J. Chem. 1968, 46, 3071-3077. [CrossRef]

13. Heinz, E.; Tulloch, A.P. Reinvestigation of the structure of acyl galactose diglyceride from spinach leaves. Hoppe Seylers Z. Physiol. Chem. 1969, 350, 493-498. [CrossRef] [PubMed]

14. Heinz, E.; Rullkötter, J.; Budzikiewicz, H. Acyl digalactosyl diglyceride from leaf homogenates. Hoppe Seylers Z. Physiol. Chem. 1974, 355, 612-616. [CrossRef] [PubMed]

15. Holmbäck, J.; Karlsson, A.A.; Arnoldsson, K.C. Characterization of N-acylphosphatidylethanolamine and acylphosphatidylglycerol in oats. Lipids 2001, 36, 153-165. [CrossRef]

16. Nilsson, A.K.; Johansson, O.N.; Fahlberg, P.; Kommuri, M.; Topel, M.; Bodin, L.J.; Sikora, P.; Modarres, M.; Ekengren, S.; Nguyen, C.T.; et al. Acylated monogalactosyl diacylglycerol: Prevalence in the plant kingdom and identification of an enzyme catalyzing galactolipid head group acylation in Arabidopsis thaliana. Plant J. 2015, 84, 1152-1166. [CrossRef] [PubMed] 
17. Murakami, N.; Shirahashi, H.; Nagatsu, A.; Sakakibara, J. Studies on glycolipids. VI. New acyl-distributed glyceroglycolipids from the nitrogen-fixing cyanobacterium Anabaena flos-aquae f. flos-aquae. Chem. Pharm. Bull. 1993, 41, 1177-1179. [CrossRef]

18. Kim, Y.H.; Choi, J.S.; Hong, J.; Yoo, J.S.; Kim, M.S. Identification of acylated glycoglycerolipids from a cyanobacterium, Synechocystis sp., by tandem mass spectrometry. Lipids 1999, 34, 847-853. [CrossRef]

19. Al-Fadhli, A.; Wahidulla, S.; D'Souza, L. Glycolipids from the red alga Chondria armata (Kutz.) Okamura. Glycobiology 2006, 16, 902-915. [CrossRef]

20. Vu, H.S.; Shiva, S.; Roth, M.R.; Tamura, P.; Zheng, L.; Li, M.; Sarowar, S.; Honey, S.; McEllhiney, D.; Hinkes, P.; et al. Lipid changes after leaf wounding in Arabidopsis thaliana: Expanded lipidomic data form the basis for lipid co-occurrence analysis. Plant J. 2014, 80, 728-743. [CrossRef] [PubMed]

21. Liedvogel, B.; Kleinig, H. Lipid metabolism in chromoplast membranes from the daffodil: Glycosylation and acylation. Planta 1977, 133, 249-253. [CrossRef] [PubMed]

22. Vu, H.S.; Tamura, P.; Galeva, N.A.; Chaturvedi, R.; Roth, M.R.; Williams, T.D.; Wang, X.; Shah, J.; Welti, R. Direct Infusion Mass spectrometry of oxylipin-containing Arabidopsis membrane lipids reveals varied patterns in different stress responses. Plant Physiol. 2012, 158, 324-339. [CrossRef] [PubMed]

23. Ibrahim, A.; Schütz, A.-L.; Galano, J.-M.; Herrfurth, C.; Feussner, K.; Durand, T.; Brodhun, F.; Feussner, I. The alphabet of galactolipids in Arabidopsis thaliana. Front. Plant Sci. 2011, 2, 95. [CrossRef]

24. Genva, M.; Andersson, M.X.; Fauconnier, M.-L. Simple liquid chromatography-electrospray ionization ion trap mass spectrometry method for the quantification of galacto-oxylipin Arabidopsides in plant samples. Sci. Rep. 2020, 10, 11957. [CrossRef]

25. Hisamatsu, Y.; Goto, N.; Hasegawa, K.; Shigemori, H. Arabidopsides A and B, Two new oxylipins from Arabidopsis thaliana. Tetrahedron Lett. 2003, 44, 5553-5556. [CrossRef]

26. Hisamatsu, Y.; Goto, N.; Sekiguchi, M.; Hasegawa, K.; Shigemori, H. Oxylipins Arabidopsides C and D from Arabidopsis thaliana. J. Nat. Prod. 2005, 68, 600-633. [CrossRef] [PubMed]

27. Buseman, C.M.; Tamura, P.; Sparks, A.A.; Baughman, E.J.; Maatta, S.; Zhao, J.; Roth, M.R.; Esch, S.W.; Shah, J.; Williams, T.D.; et al. Wounding stimulates the accumulation of glycerolipids containing oxophytodienoic acid and dinor-oxophytodienoic acid in Arabidopsis leaves. Plant Physiol. 2006, 142, 28-39. [CrossRef]

28. Shiva, S.; Samarakoon, T.; Lowe, K.A.; Roach, C.; Vu, H.S.; Colter, M.; Porras, H.; Hwang, C.; Roth, M.R.; Tamura, P.; et al. Leaf lipid alterations in response to heat stress of Arabidopsis thaliana. Plants 2020, 9, 845. [CrossRef]

29. Mongrand, S.; Bessoule, J.-J.; Cabantous, F.; Cassagne, C. The C16:3 \C18:3 fatty acid balance in photosynthetic tissues from 468 plant species. Phytochemistry 1998, 49, 1049-1064. [CrossRef]

30. Hartley, S.E.; Eschen, R.; Horwood, J.M.; Gange, A.C.; Hill, E.M. Infection by a foliar endophyte elicits novel arabidopside-based plant defence reactions in its host, Cirsium arvense. New Phytol. 2015, 205, 816-827. [CrossRef]

31. Ohashi, T.; Ito, Y.; Okada, M.; Sakagami, Y. Isolation and stomatal opening activity of two oxylipins from Ipomoea tricolor. Bioorganic Med. Chem. Lett. 2005, 15, 263-265. [CrossRef] [PubMed]

32. Zábranská, M.; Vrkoslav, V.; Sobotníková, J.; Cvačka, J. Analysis of plant galactolipids by reversed-phase high-performance liquid chromatography/mass spectrometry with accurate mass measurement. Chem. Phys. Lipids 2012, 165, 601-607. [CrossRef] [PubMed]

33. Mueller, S.P.; Unger, M.; Guender, L.; Fekete, A.; Mueller, M.J. Phospholipid:diacylglycerol acyltransferase-mediated triacylglyerol synthesis augments basal thermotolerance. Plant Physiol. 2017, 175, 486-497. [CrossRef] [PubMed]

34. Heinz, E. On the enzymatic formation of acylgalactosyl diglyceride. Biochim. Biophys. Acta 1967, 144, 333-343. [CrossRef]

35. Heinz, E.; Dieler, H.P.; Rullkötter, J. Enzymatic acylation of steryl glycoside. Z. Pflanzenphysiol. 1975, 75, 78-87. [CrossRef]

36. Heinz, E. Some properties of the acyl galactose diglyceride-forming enzyme from leaves. Z. Pflanzenphysiol. 1973, 69, 359-376. [CrossRef]

37. Heinz, E.; Betrams, M.; Joyard, J.; Douce, R. Demonstration of an acyltransferase activity in chloroplast envelopes. Z. Pflanzenphysiol. 1978, 87, 325-331. [CrossRef]

38. Heemskerk, J.W.M.; Wintermans, J.F.G.M.; Joyard, J.; Block, M.A.; Dorne, A.; Douce, R. Localization of galactolipid: Galactolipid galactosyltransferase and acyltransferase in outer envelope membrane of spinach chloroplasts. Biochim. Biophys. Acta 1986, 877, 281-289. [CrossRef]

39. Nilsson, A.K.; Fahlberg, P.; Ellerström, M.; Andersson, M.X. Oxo-phytodienoic acid (OPDA) is formed on fatty acids esterified to galactolipids after tissue disruption in Arabidopsis thaliana. FEBS Lett. 2012, 586, 2483-2487. [CrossRef]

40. Froehlich, J.E.; Itoh, A.; Howe, G.A. Tomato allene oxide synthase and fatty acid hydroperoxide lyase, two cytochrome P450s involved in oxylipin metabolism, are targeted to different membranes of chloroplast envelope. Plant Physiol. 2001, 125, 306-317. [CrossRef]

41. Farmaki, T.; Sanmartín, M.; Jiménez, P.; Paneque, M.; Sanz, C.; Vancanneyt, G.; León, J.; Sánchez-Serrano, J.J. Differential distribution of the lipoxygenase pathway enzymes within potato chloroplasts. J. Exp. Bot. 2007, 58, 555-568. [CrossRef]

42. Bräutigam, A.; Hoffmann-Benning, S.; Weber, A.P.M. Comparative proteomics of chloroplast envelopes from C3 and C4 plants reveals specific adaptations of the plastid envelope to $\mathrm{C} 4$ photosynthesis and candidate proteins required for maintaining $\mathrm{C} 4$ metabolite fluxes. Plant Physiol. 2008, 148, 568-579. [CrossRef]

43. Schaller, F.; Zerbe, P.; Reinbothe, S.; Reinbothe, C.; Hofmann, E.; Pollmann, S. The allene oxide cyclase family of Arabidopsis thaliana-Localization and cyclization. FEBS J. 2008, 275, 2428-2441. [CrossRef] [PubMed] 
44. Peltier, J.-B.; Ytterberg, A.J.; Sun, Q.; van Wijk, K.J. New functions of the thylakoid membrane proteome of Arabidopsis thaliana revealed by a simple, fast, and versatile fractionation strategy. J. Biol. Chem. 2004, 279, 49367-49383. [CrossRef]

45. Vidi, P.-A.; Kanwischer, M.; Baginsky, S.; Austin, J.R.; Csucs, G.; Dörmann, P.; Kessler, F.; Bréhélin, C. Tocopherol cyclase (VTE1) localization and vitamin E accumulation in chloroplast plastoglobule lipoprotein particles. J. Biol. Chem. 2006, 281, 11225-11234. [CrossRef] [PubMed]

46. Swamy, M.J.; Ramakrishnan, M.; Angerstein, B.; Marsh, D. Spin-label electron spin resonance studies on the mode of anchoring and vertical location of the $\mathrm{N}$-acyl chain in N-acylphosphatidylethanolamines. Biochemistry 2000, 39, 12476-12484. [CrossRef]

47. Swamy, M.J.; Tarafdar, P.K.; Kamlekar, R.K. Structure, phase behaviour and membrane interactions of N-acylethanolamines and N-acylphosphatidylethanolamines. Chem. Phys. Lipids 2010, 163, 266-279. [CrossRef] [PubMed] 